\title{
Régulation et pilotage des systèmes scolaires: les voies de l'harmonisation dans deux systèmes scolaires historiquement décentralisés, l'Angleterre et la Belgique francophone
}

\section{Vincent Dupriez}

Les systèmes scolaires d'Angleterre et de Belgique francophone ont tous deux connu au cours des 15 dernières années un important processus d'harmonisation et de standardisation. La comparaison de ces deux systèmes fait apparaître à quel point ces transformations se réalisent dans des environnements institutionnels contrastés et avec de très nettes différences dans le recours à l'évaluation comme ressource pour le pilotage. Dans les deux situations cependant, la montée en puissance de référents nationaux de nature prescriptive, contribuant à l'harmonisation des systèmes scolaires, semble heurter la légitimité professionnelle des enseignants et la place de leur expertise comme source d'influence et de reconnaissance.

A la croisée de la sociologie des organisations, des sciences politiques et des sciences de l'éducation, les questions de régulation et de pilotage des systèmes scolaires renvoient à l'analyse de la coordination de l'action dans le champ scolaire et en particulier aux modes de construction et de contrôle de l'action éducative. Un postulat fondamental repose derrière la définition d'un tel objet de recherche, c'est celui d'une relative stabilité et d'une relative autonomie des formes sociales. Et, comme le relève Norbert Alter (2001), ces formes sociales ne sont pas seulement des règles: «Elles correspondent également aux dimensions culturelles des relations, à ce qui les stabilise. Ces formes consistent à établir de manière durable, prévisible et connue par tous des pratiques de travail, des relations de travail ou des modalités de jugement sur l'activité» (Alter 2001, p. 156). L'analyse de la régulation passe dès lors par le repérage des différentes formes sociales qui contribuent à orienter et à stabiliser les conduites des acteurs.

Dans ce sens, Maroy et Dupriez (2000) et Dupriez et Maroy (2003) ont défini la régulation comme «le processus de production de règles et d'orientation des conduites des acteurs dans un espace social déterminé» (Maroy et Dupriez 2000, p. 73). Au regard de cette définition très large de la régulation, ne sera prise en considération dans ce texte qu'une seule source de régulation: celle qui relève des pouvoirs publics et est parfois qualifiée de pilotage (De Landsheere 1994) ou encore de gouvernance. Une telle analyse fait donc globalement l'im- 
passe sur de nombreuses autres sources de régulation et en particulier sur la contribution des professionnels de l'éducation à la construction d'une action pédagogique locale, plus ou moins distante des projets déclarés des responsables éducatifs. L'analyse proposée dans ce texte fera cependant apparaître à quel point des modèles généraux de pilotage (ou de gouvernance) prennent des formes spécifiques en s'adaptant à l'histoire des système scolaires et aux configurations d'acteurs diversement présentes dans les pays analysés.

La plupart des analyses portant sur le pilotage des systèmes scolaires se rejoignent pour affirmer qu'il existe un renouvellement des modes de régulation accordant aujourd'hui davantage de place à l'évaluation d'une part, et à des ajustements locaux entre l'offre et la demande d'éducation d'autre part. Ces transformations adoptent des voies diverses en fonction des pays considérés et de l'histoire des systèmes scolaires. Il est cependant légitime de se référer à un certain nombre de schémas interprétatifs communs qui, au-delà de la situation singulière de chaque système, aident à comprendre la nature des transformations observées. Un premier objectif de ce texte est de proposer certains de ces schémas afin de pouvoir s'appuyer sur des référents conceptuels explicites pour analyser l'évolution des systèmes scolaires. Un second objectif est d'étudier, à partir de ces schémas, l'évolution de la régulation scolaire dans deux systèmes scolaires historiquement décentralisés, celui de la Belgique francophone et celui de l'Angleterre, en faisant apparaître les logiques d'intervention, historiquement situées, mobilisées par les autorités publiques.

\section{Le pilotage comme mode de construction d'une représentation de la réalité}

A la suite de Jobert (1992), il est important de rappeler à quel point toute action sociale, et en particulier toute action publique, participe d'un travail de définition de la réalité. Autrement dit, le mode de présentation d'une politique publique, le repérage d'éléments apparaissant comme pertinents ou marginaux et les modes d'interprétation de la réalité suggérés contribuent à rendre légitime un mode de perception de celle-ci. L'action publique, au-delà des objectifs explicites qu'elle entend promouvoir, s'inscrit dès lors dans un processus de modélisation de la réalité sociale. Aux yeux de Jobert (1992), les référentiels des politiques publiques, entendus comme des ensembles de croyances, de valeurs et de techniques qui structurent la scène des politiques publiques, combinent trois dimensions permettant de saisir leur contribution à ce travail de mise en forme de la réalité. La première dimension est cognitive et suggère que toute politique publique est constitutive d'une sélection de faits significatifs, d'un mode de définition de ces faits et d'un schéma de relations entre ces faits. Elle affecte donc la manière même d'appréhender et de penser un phénomène. La seconde dimension est normative. A ce titre, les politiques publiques sont porteuses de valeurs 
et affectent la vision de ce qui est souhaitable et de ce qui ne l'est pas. Enfin, les politiques publiques et les référentiels auxquels elles se rattachent définissent des principes d'action (dimension instrumentale) fondés sur ces savoirs et sur ces valeurs.

Il importe donc de prendre en compte, au-delà des objectifs déclarés de toute politique publique, sa contribution à une transformation de l'espace social et des catégories qui permettent de penser cet espace. A ce titre par exemple, il est évident que l'émergence des notions de standards, d'usagers, d'accountability, ont un impact non négligeable sur la définition de ce qui est pertinent et de ce qui l'est moins dans l'espace scolaire. Avec Derouet (1992), on peut considérer que ces notions inscrivent l'école au croisement des mondes industriel et marchand, voire civique. Elles rendent légitime les préoccupations d'efficience et d'efficacité et soustraient l'école d'un monde domestique où les qualités du travail enseignant ne sont par définition ni mesurables ni évaluables. Au-delà donc des changements les plus explicites, il importe de prendre en compte la transformation plus latente des représentations sociales du monde scolaire qui accompagnent les évolutions dans le pilotage de l'école.

\section{Vers un Etat évaluateur}

Pour comprendre l'évolution des politiques publiques en éducation, le référentiel de l'Etat évaluateur (Neave 1989) est indéniablement d'une certaine utilité. Ce concept résume de manière assez heureuse diverses transformations et met en évidence un certain type de (re)distribution des fonctions entre le centre et la périphérie. Dans l'esprit de ce référentiel, les autorités politiques, au centre, se chargent de définir les objectifs à atteindre et garantissent une forme de contrôle et d'évaluation du travail de chacune des unités décentralisées (les établissements, généralement). Parallèlement, chacun des établissements ou des groupes d'établissements bénéficie de plus de liberté dans la définition de son projet, mais doit rendre des comptes aux autorités politiques, au regard des objectifs qui lui ont été assignés. Pour Neave toujours, suivi par Van Haecht (1998), cette progressive redistribution des fonctions entre le centre et la périphérie est telle qu'elle est susceptible de dépasser - et de rendre caduque - l'opposition classique entre systèmes centralisés et décentralisés. Dans les pays à tradition centralisée comme la France, des changements récents reconnaissent aux autorités régionales ou départementales d'importantes responsabilités en matière de construction et d'équipements scolaires, et aux établissements de nouvelles responsabilités relatives aux choix pédagogiques et à l'usage de la dotation horaire. A partir d'épreuves standardisées des acquis scolaires des élèves, les établissements sont par ailleurs informés - et parfois, interpellés - en fonction de leur capacité à faire progresser leurs élèves. Dans les systèmes à tradition davantage décentralisée comme l'Angleterre, les réformes adoptées depuis la fin des années 80 ont notamment intro- 
duit un curriculum national et des évaluations externes standardisées des élèves à quatre reprises durant la scolarité obligatoire. Il y a donc dans ces deux pays une redistribution des fonctions entre le centre et la périphérie avec un centre politique prioritairement concentré sur la définition d'objectifs et d'indicateurs servant à mesurer le travail des unités décentralisées, ces dernières bénéficiant de marges de liberté - variables, selon les pays - leur permettant de construire des stratégies propres pour atteindre les objectifs définis. Notons dès à présent que, derrière cette apparente similitude, il y a cependant des logiques d'action qui ne peuvent être confondues. On peut en effet avec Maroy (2004) distinguer une logique de contractualisation d'une logique de quasi-marché. Dans le premier cas, la source d'interpellation est l'autorité publique qui, à travers un corps d'inspecteurs ou d'agents intermédiaires, fait pression sur les établissements tenus pour responsables des résultats obtenus. Dans la seconde logique, le rôle de l'autorité publique est davantage d'informer les clients scolaires en diffusant des informations relatives à la qualité des établissements. La pression sur la qualité est censée trouver ici son origine dans le comportement de parents avertis, susceptibles de pousser les établissements vers une amélioration de leurs pratiques. Dans les deux cas, il y a bien une logique de pilotage par les résultats, mais la configuration d'acteurs et la source de pression sont clairement distinctes.

Mais, si cette référence à l'Etat évaluateur permet de clarifier le débat, elle reste par ailleurs trop grossière pour rendre compte de la diversité et de la complexité des situations analysées. La référence à une pluralité de formes de coordination, proposée par Maroy et Dupriez (2000) et Dupriez et al. (2003) permet à ce titre de dépasser une analyse trop centrée sur l'opposition centre-périphérie. C'est également une proposition qui permet de dépasser le traditionnel clivage de la littérature économique, la régulation par l'Etat ou par le Marché.

\section{Pluralité des formes de coordination}

En référence au monde socio-économique, Hollingsworth et Boyer (1997) ont proposé une typologie des formes de coordination qui permet de rendre compte de la diversité des arrangements institutionnels susceptibles de construire de la coordination entre acteurs de la vie socio-économique. Cette typologie propose en fait une pluralité de logiques d'actions qui peuvent être situées au regard de deux axes. L'axe horizontal renvoie au ressort de l'action et oppose les contextes où l'acteur est appréhendé comme un individu stratégique mû par la défense de ses intérêts aux contextes où l'individu est perçu comme membre d'une collectivité, lié aux autres par des valeurs communes et des obligations réciproques. L'axe vertical correspond à la distribution du pouvoir et permet de différencier des situations où le pouvoir est distribué sur une base hiérarchique (coordination par imposition) de situations où les personnes s'engagent de manière plus volontaire dans des rapports d'échanges (coordination par implication). 


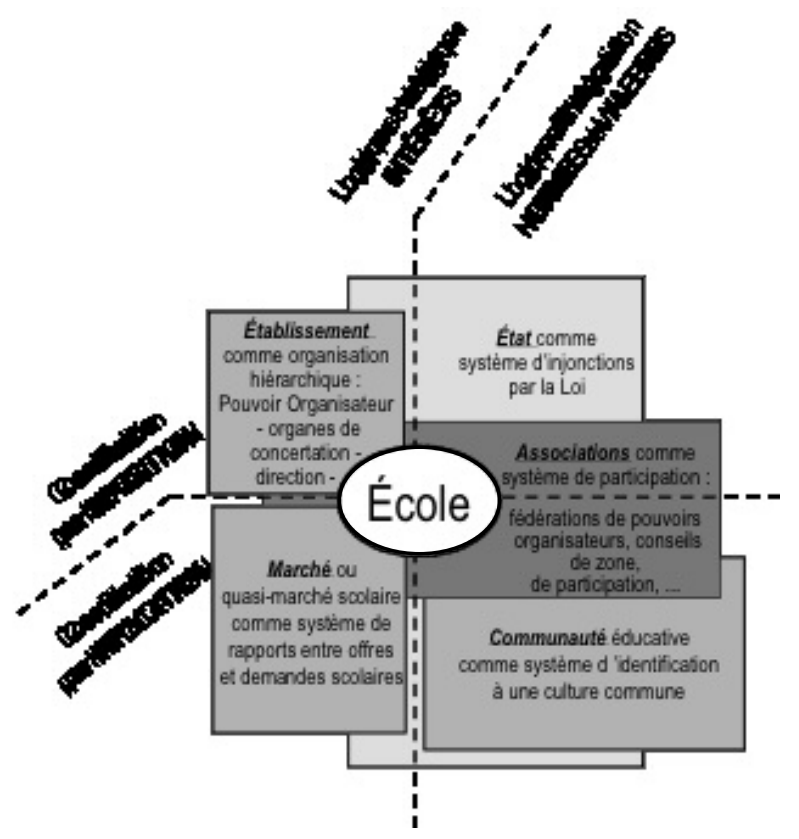

Figure 1: Les formes de coordination dans le système scolaire

Ces deux axes correspondent évidemment à des continuums et la position de chacune des formes de coordination doit être prise à titre indicatif. Dans la figure 1 , nous proposons une adaptation de la typologie de Hollingsworth et Boyer en fonction des spécificités du champ scolaire, tout en restant fidèle aux formes de coordination suggérées par ces auteurs. L'Etat correspond à l'autorité publique qui, bénéficiant de la légitimité issue du scrutin, peut définir et imposer ce qui semble correspondre à l'intérêt général. Son mode d'intervention passe principalement par la Loi. Les associations renvoient à un système de participation volontaire entre des acteurs individuels et/ou collectifs. Les acteurs y sont liés de manière souple et veillent généralement à construire ou à défendre un intérêt commun, comme dans les syndicats ou les fédérations d'établissements par exemple. Dans les communautés, les acteurs entretiennent des relations basées sur la réciprocité, la confiance et les intérêts communs du groupe. Une culture commune est généralement une source puissante d'intégration. La logique de marché correspond à un système d'échange volontaire entre des transactants autonomes, veillant à faire valoir leurs intérêts propres. Dans le monde scolaire, les économistes se réferent à la notion de quasi-marché (Vandenberghe 1999) pour caractériser les systèmes scolaires où, à partir d'un financement public, les familles peuvent choisir leur établissement, financé en fonction du nombre d'élèves inscrits. Enfin, l'établissement ${ }^{1}$ peut être analysé comme une organisa- 
tion où, autour d'une ligne hiérarchique (Conseil d'administration, chef d'établissement, ...), des acteurs individuels et collectifs négocient leurs positions et tâches respectives.

Dans toutes les situations que nous avons pu étudier jusqu'à présent, différentes logiques opèrent simultanément au sein d'un système scolaire et les acteurs se positionnent, en fonction du contexte de leurs interactions, au regard de ces différents arrangements institutionnels. Ainsi, l'histoire du système scolaire en Angleterre repose sur une très large autonomie des autorités locales (Local Education Authorities) libres de définir notamment les programmes d'étude et les épreuves d'évaluation des élèves. Cette autonomie des autorités locales se conjuguait avec un système de carte scolaire, imposant aux familles de fréquenter l'école de leur quartier. Les parents ne pouvaient donc pas choisir entre des écoles offrant des services inégaux. C'est la dénonciation de cette situation, couplée à des interrogations sur l'efficacité du système, qui a conduit aux réformes de 1988 (Education Reform Act). Ces dernières apparaissent prioritairement comme la construction volontaire d'un quasi-marché scolaire, fondé sur le slogan de la liberté de choix de l'école par les familles. Mais, au-delà de la référence au marché, il importe de prendre en compte le renforcement paradoxal de l'Etat qui semble nécessaire au bon fonctionnement du marché. C'est ainsi en 1988 qu'est créé pour la première fois en Angleterre un curriculum national et que se développent progressivement le recours à des batteries d'épreuves externes destinées à évaluer les compétences des élèves. Dans ce sens, on peut considérer que l'harmonisation du système scolaire en Angleterre n'était sans doute pas un objectif en soi, mais est apparue comme une condition pour la construction du quasi-marché: il était nécessaire de standardiser un minimum les services éducatifs et les critères d'évaluation de ceux-ci afin de permettre aux consommateurs de raisonner sur une base comparative. Cela s'est fait tout en valorisant, d'un point de vue rhétorique $\mathrm{du}$ moins, l'autonomie des établissements et en ne touchant que modestement aux liens étroits d'insertion de certaines écoles dans une communauté locale.

Cette typologie des formes de coordination est également très éclairante pour comprendre les règles du jeu scolaire en Belgique francophone ${ }^{2}$ (cf. Maroy $\&$ Dupriez, 2000). Le système scolaire belge est historiquement très décentralisé avec une large autonomie accordée à chacun des pouvoirs organisateurs. Ces derniers sont entre autres responsables de l'engagement du personnel, des programmes d'étude, de la définition des orientations pédagogiques et de l'évaluation des élèves dans l'(es) établissement (s) placé(s) sous leur responsabilité. Ces pouvoirs organisateurs sont tantôt privés (et généralement liés à des organisations catholiques), tantôt publics (et dépendent soit du pouvoir central, soit des autorités politiques locales telles que les communes). Historiquement, cette autonomie de chaque pouvoir organisateur s'explique par des conflits d'ordre philosophique (entre milieux laïques et catholiques). Le compromis adopté en Belgique reconnaît la liberté des familles à pouvoir choisir entre un enseignement confessionnel ou non-confessionnel et affirme également la liberté des écoles à 
proposer leur propre projet de formation. Mais cette liberté, fondée initialement sur un argument philosophique, est clairement devenue l'objet d'autres enjeux: les écoles se font aujourd'hui concurrence pour capter un public, de moins en moins soucieux du caractère confessionnel ou non de l'établissement. L'histoire du quasi-marché scolaire observé en Belgique est donc clairement distincte de la situation en Angleterre. Dans ce contexte de très large décentralisation, et face aux très importantes inégalités de résultats observées en particulier en Belgique francophone, les autorités politiques ont pris récemment de nombreuses initiatives tendant à formuler un ensemble de règles s'appliquant à tous les pouvoirs organisateurs. C'est à ce titre notamment que depuis 1997, des référentiels de compétences (objectifs de formation, définis en termes de compétences à faire acquérir aux élèves) sont définis et s'appliquent progressivement à tous les niveaux d'étude. Dorénavant, tout pouvoir organisateur souhaitant travailler à partir de son propre programme doit au préalable faire la preuve que son programme correspond aux référentiels de compétences. Une telle initiative a pu être adoptée, malgré le fait qu'elle heurte le principe constitutionnel de liberté d'enseignement, dans la mesure où elle a fait l'objet de très longues concertations entre les représentants de l'autorité publique et les puissants représentants des fédérations d'établissements. Ce n'est donc pas un Etat tout puissant qui redéfinit les règles du jeu, mais plutôt un lent travail de compromis à construire entre les acteurs les plus influents. Parallèlement à cette montée en puissance tant du quasimarché scolaire que du recours à la loi pour coordonner l'action scolaire, l'autonomie des acteurs locaux et en particulier des pouvoirs organisateurs reste importante. C'est au regard de cette complexité et de cette absence d'une logique dominante que Maroy et Dupriez (2000) parlent de montage composite pour qualifier l'état des formes de coordination dans le système scolaire en Belgique francophone.

\section{Place et formes de L'évaluation en Belgique et en Angleterre}

En Belgique francophone comme en Angleterre, on observe donc des autorités politiques centrales qui affirment leur présence avec davantage de force, en s'appuyant principalement sur un travail de standardisation des objectifs d'enseignement. Dans ces deux systèmes scolaires, on observe également une puissante logique de quasi-marché scolaire (dont l'émergence et la signification sont cependant clairement différentes) et une autonomie non-négligeable des acteurs locaux. Une différence majeure au niveau de l'évolution de la régulation dans ces deux systèmes correspond à la place et aux caractéristiques de l'évaluation. 


\section{En Angleterre}

Il faut distinguer en Angleterre deux dispositifs complémentaires d'évaluation des élèves et des établissements. D’une part, la réforme éducative de 1988 a introduit un programme national d'évaluation, s'appuyant sur des mesures standardisées des apprentissages aux âges de 7, 11, 14 et 16 ans. Ces évaluations externes nationales viennent par ailleurs souvent s'ajouter à d'autres épreuves standardisées que mettent en place certaines autorités locales. L'objectif des ces évaluations est d'une part d'informer les élèves et les parents de leur niveau scolaire, mais aussi d'apporter une information permettant de juger de la qualité des enseignants et des établissements. Cette évaluation des établissements s'appuie parallèlement sur le travail d'équipes d'inspecteurs privés recrutés par l'Office for standards in Education (OFSTED). Ces équipes d'inspecteurs sont chargées de l'inspection de l'ensemble des écoles primaires et secondaires sur base de critères communs. Pour réaliser ces évaluations, les inspecteurs mènent un travail d'observation et de rencontre avec les différents acteurs de l'école, y compris les parents. Le niveau des élèves dans l'établissement représente bien entendu une des principales rubriques de cette évaluation. Mais celle-ci inclut également une appréciation sur la qualité de l'offre éducative, sur le développement spirituel, moral, social et culturel des élèves et sur l'utilisation des ressources financières (van Zanten 2001).

Le rapport final de cette évaluation est transmis au Conseil d'Administration de chaque établissement qui est invité à y réagir à travers un plan d'action et d'objectifs précis. Le rapport est par ailleurs rendu public et peut être consulté via Internet. Un résumé du rapport est spécialement conçu pour les parents. Ces inspections et en particulier les informations apportées sur le niveau des élèves représentent un enjeu considérable pour les écoles, dans la mesure où les parents en prennent connaissance et sont invités à en tenir compte pour le choix d'une école. Par ailleurs, comme le relève Broadfoot, «Les résultats des examens publics et des évaluations nationales qui fournissent les éléments statistiques sont souvent interprétés sans distance et hors de leur contexte. L'utilisation abusive des données brutes pour comparer certains établissements est évidemment injuste pour les enseignants qui travaillent dans les établissements difficiles» (Broadfoot 2000 , p. 52). Dans le but d'éviter des interprétations déplacées de la qualité des établissements, l'OFSTED s'appuie bien à certains moments sur des comparaisons entre écoles au public semblable, mais ce ne sont pas ces indices qui apparaissent dans les League tables, ni qui retiennent l'attention des parents.

A la suite de Broadfoot $(1996,2000)$, on peut considérer que le système anglais s'inscrit aujourd'hui dans une perspective managériale axée sur la performance. Inscrite dans une logique d'accountability, la conjonction d'un curriculum national, d'un système d'évaluation standardisé et d'un dispositif de diffusion de l'information produit incontestablement des effets. Il semble notamment que le niveau moyen de l'éducation en Angleterre ait évolué positive- 
ment avec ces réformes. Ce gain en efficacité semble par contre s'accompagner d'une perte en équité et d'un accroissement des écarts de réussite entre établissements. Mais, de manière parallèle, Broadfoot insiste sur la puissance normalisatrice d'un tel dispositif et sur sa capacité à affecter les structures cognitives et les conduites des acteurs: "Toutes ces mesures conditionnent le discours sur l'éducation. Il est presque impossible pour un établissement ou un enseignant de s'opposer à une forme d'évaluation qui permet d'établir les jugements sur le travail de chaque enseignant, celui des autorités locales et, en fin de compte, la qualité du système dans son ensemble» (Broadfoot, 2000, p. 51). En référence à Jobert (1992), on peut considérer que le dispositif d'évaluation, derrière une technologie apparemment neutre, apparaît comme un puissant vecteur de construction cognitive et normative, affectant la définition de ce qui est visible et de ce qui compte dans le champ scolaire.

Notons encore à propos des dispositifs d'évaluation en Angleterre que l'information issue du travail de mesure des acquis des élèves alimente un double processus. D'une part, le niveau moyen des élèves de l'école est une des rubriques du rapport de l'OFSTED et, à ce titre, c'est bien à l'Etat que l'établissement doit rendre des comptes. En fonction du rapport d'évaluation, l'Etat n'interviendra d'ailleurs que très peu si les résultats sont satisfaisants; des mesures de pression et éventuellement de sanction seront par contre adoptées si les résultats sont insatisfaisants. D'autre part, les résultats des établissements sont communiqués aux parents d'élèves et ceux-ci sont invités à les prendre en compte pour choisir l'école de leur enfant. Dans les deux cas, les mesures d'acquis scolaires des élèves représentent la source d'information. Mais, dans la première situation, cette information est communiquée aux autorités publiques qui exercent alors directement une pression sur les établissements dont les résultats sont considérés comme insuffisants. On est donc bien en présence ici d'un pilotage par les résultats inscrit dans une logique de contractualisation. Dans la seconde situation, la pression potentielle est exercée par les familles: on est dans la logique du quasimarché.

\section{En Belgique francophone}

Le système scolaire en Belgique se caractérise également par une situation historique de décentralisation. Au nom du principe constitutionnel de liberté d'enseignement, les parents peuvent choisir l'école de leur enfant et les écoles sont libres de définir leur propre projet pédagogique. Prenant conscience de la grande disparité de pratiques d'enseignement, des énormes inégalités de résultats entre élèves et de la faible efficacité du système scolaire (Crahay 1997; Vandenberghe 2002), des dispositions ont cependant été prises au cours des dernières années dans le but de rendre le système scolaire plus homogène. La diffusion, sous forme de référentiels de compétences, d'objectifs de formation communs pour tous les 
élèves en représente une pièce non négligeable et peut être considérée comme une condition nécessaire à un pilotage par les résultats. Des mesures parallèles ont également été adoptées visant notamment à rapprocher les statuts des personnels enseignants (malgré la différence de nature des pouvoirs organisateurs entre l'école publique et l'école catholique), à imposer des modes de fonctionnement au sein des établissements (en rendant, par exemple, la concertation entre enseignants obligatoire) ou encore en définissant par décret ce qui est souhaitable en matière d'orientations pédagogiques. Cette tendance à l'harmonisation du système scolaire et cette définition d'objectifs de formation communs peuvent être comprises comme un travail préalable, comme une sorte de pré-requis à un modèle de régulation s'appuyant sur les résultats. Mais, les mesures d'harmonisation pédagogique et organisationnelle illustrent également l'ambiguïté présente en Belgique francophone par rapport à la question du pilotage: parallèlement à l'ébauche d'un pilotage par les résultats, les autorités adoptent des mesures prescriptives, reposant davantage sur une rationalité bureaucratique.

On observe par ailleurs en Belgique francophone une faiblesse manifeste des dispositifs d'évaluation externe, qui peut être illustrée par deux exemples.

1. Dans l'enseignement primaire et secondaire, depuis le début des années nonante, une évaluation externe a été mise en place. Ces épreuves sont organisées tous les deux ans en début d'année scolaire, à chaque fois pour une année d'étude différente. Seuls sont traités les résultats d'un échantillon représentatif des établissements (généralement, 2 classes par établissement) afin de produire une moyenne de l'état des apprentissages en Belgique francophone. Les autres établissements intéressés par l'évaluation s'inscrivent sur une base volontaire. Les résultats de l'échantillon sont utilisés d'une part pour mieux connaître l'état global du système scolaire et d'autre part pour permettre à chaque classe ou établissement, volontaire ou faisant partie de l'échantillon, de se situer par rapport à une moyenne générale. Ces résultats sont communiqués aux écoles, accompagnés de pistes didactiques, c'est-à-dire de suggestions d'activités en lien avec chaque partie de l'épreuve. Mais, à aucun moment, il n'est prévu ni de rendre public les résultats des écoles, ni de classer les écoles en fonction de leurs scores. En d'autres mots, cette épreuve externe a fondamentalement une valeur formative, dans le sens qu' elle apporte une information (partielle et confidentielle) permettant aux enseignants de prendre du recul par rapport à l'efficacité de leur travail.

2. En lien avec les référentiels de compétences évoqués ci-dessus, le gouvernement a prévu, dès 1997, de produire des batteries d'épreuves étalonnées aux compétences requises, à différents paliers de la formation. L'absence de ces épreuves, 7 ans après l'approbation du décret, est déjà en soi révélatrice de la difficulté du système scolaire à assumer une régulation par les résultats. Mais, plus fondamentalement, c'est la place même de ces épreuves dans le dispositif 
global qui révèle une forme très modeste et très locale de l'Etat évaluateur. Il n'est jusqu’à présent pas prévu de faire appliquer ces épreuves dans toutes les classes. Ces épreuves visent plutôt à proposer un étalon de ce qui peut être exigé d'un élève à un moment de sa scolarité. Et la capacité d'influencer les pratiques pédagogiques à travers la diffusion de telles épreuves dépend de la volonté et de la force d'interpellation des deux catégories d'acteurs (l'inspection et les parents) autorisés à invoquer cet étalon pour contester la justesse des épreuves utilisées en classe par les enseignants.

Cet usage plutôt original des outils d'évaluation ne doit pas être compris comme la marque d'une irrationalité des décideurs (Dupriez \& Maroy 2000), mais plutôt comme un développement idiosyncrasique à lire à la lueur de la complexité effective des formes de coordination dans ce système scolaire. Il est notamment révélateur d'un impératif de respect de la liberté de chaque pouvoir organisateur et, simultanément, de la peur d'accentuer la concurrence entre écoles ni assumée, ni souhaitée par la majorité des acteurs scolaires. Cet usage particulier des épreuves d'évaluation n'est donc pas un accident de parcours, mais est au contraire une puissante illustration de l'ancrage spécifique de l'Etat évaluateur, eu égard aux spécificités des formes de coordination qui prévalent ici. Stricto sensu, il convient d'ailleurs de reconnaître qu'il n'y a pas aujourd'hui en Belgique francophone de pilotage par les résultats, mais plutôt une lente transformation du système scolaire visant à rendre possible un tel mode de gouvernance.

\section{Conclusion: diverses sources de légitimité en concurrence}

L'analyse présentée jusqu’à présent fait apparaître comment deux systèmes scolaires de tradition décentralisée ont assumé différemment leurs évolutions récentes conduisant de part et d'autre à une relative harmonisation du système scolaire. S'appuyant explicitement sur une idéologie de quasi-marché, l'Angleterre a adopté des transformations qui prétendent simultanément donner plus de liberté aux familles et améliorer l'efficacité du système scolaire. Avec Broadfoot notamment, il importe de prendre conscience des effets collatéraux d'une telle politique. Cette auteure insiste tout particulièrement sur les effets de normalisation liés à un tel mode de pilotage: l'imposition d'un mode de penser et d'agir qu'il devient difficile de contester au regard de l'omniprésence des références à l'évaluation. D'autres auteurs s'inquiètent plutôt des effets spécifiques du quasimarché et de sa contribution à un accroissement des inégalités entre élèves et entre établissements (Noden 2000; Gibson \& Ashtana 1999).

Mais, en s'appuyant sur une contribution de Pelletier (2001), il nous semble important de clore cette analyse du système scolaire en Angleterre par une réflexion sur la diversité des sources de légitimité dans le monde de l'école. Pelle- 
tier (2001) distingue trois sources de légitimité potentiellement concurrentes au sein des systèmes scolaires. La première est la légitimité démocratique. C'est la légitimité qui entoure toute prescription émanant des autorités politiques. La légitimité d'une réforme par exemple repose sur la légitimité démocratique de ceux qui l'ont adoptée: c'est au regard d'un mandat de représentation du peuple que les élus politiques détiennent un droit d'orientation et d'intervention dans le système scolaire. La seconde source de légitimité, c'est la légitimité de marché. Est ici légitime ce qui est susceptible d'emporter l'adhésion des parents-consommateurs d'école. C'est légitime parce que cela a du succès. Notons que cette forme de légitimité peut être observée dans des environnements différents du quasi-marché, où les familles ne choisissent pas leur école, même si son expression adoptera peut-être alors d'autres formes. Enfin, la troisième source de légitimité c'est l'expertise professionnelle des enseignants (ou de manière plus large des personnels de l'éducation). Ces trois sources de légitimité sont potentiellement concurrentes et peuvent être opposées dans des conflits relatifs à l'orientation des systèmes scolaires. Ces trois sources de légitimité occupent par ailleurs des places différentes en fonction des systèmes scolaires considérés.

Dans cette perspective, la critique majeure adressée aujourd'hui au mode de gouvernance du système scolaire en Angleterre, c'est précisément d'avoir mis à mal la légitimité professionnelle du corps enseignant, au profit d'une légitimité techno-démocratique (les standards imposés par les autorités politiques) et d'une légitimité marchande (la nécessité de plaire aux parents pour garder leur enfant dans l'école). A ce titre, Wrigley (2003) attire l'attention sur la tendance à la déprofessionalisation du corps enseignant qui est soumis à une rationalité technomarchande, sur base des critère retenus pour l'évaluation des élèves et des établissements. Clarke (2001) souligne également les conséquences néfastes d'une culture de la performance qui heurte de front les formes d'engagement moral des enseignants. van Zanten (2001) insiste pour sa part sur le travail des équipes d'inspection qui met les enseignants en "position d'exécutants devant se soumettre à un regard normatif élaboré à l'extérieur» (van Zanten 2001, p. 129). Broadfoot (2000) enfin relève qu'autrefois, il suffisait de faire état de son statut professionnel comme base légitime de son autonomie. Aujourd'hui, il faut surtout faire la preuve de sa compétence professionnelle, appréhendée le plus souvent à travers la mesure étroite des standards de formation. En bref, le puissant investissement dans la construction d'un quasi-marché scolaire, appuyé sur la définition de références communes à l'aune desquelles les élèves et les écoles sont évalués, s'est clairement fait au détriment de la reconnaissance et du développement de l'expertise des enseignants.

Même si en Belgique francophone, la question se pose différemment, elle n'en est pas moins préoccupante au regard de cette interrogation sur la place de la légitimité professionnelle des personnels de l'éducation. Commençons par rappeler que même si le référentiel de l'Etat évaluateur a sans doute orienté diverses décisions politiques récentes, on ne peut pas parler aujourd'hui d'un pilotage par 
les résultats en Belgique francophone ${ }^{3}$. Au contraire, comme cela a été documenté ailleurs (Draelants, Dupriez et Maroy, 2003), les prémisses d'un pilotage par les résultats s'accompagnent d'une série de mesures récentes de type bureaucratiques où l'autorité publique, davantage que précédemment, définit ce qui est attendu des acteurs scolaires en termes de fonctionnement d'établissement (obligation de concertation entre enseignants, de rédaction d'un projet d'établissement, de mise en place d'un Conseil de participation, p. ex.) et en termes d'orientations pédagogiques (prescription de l'évaluation formative et de la pédagogie différenciée).

Malgré cette absence totale d'une pression sur les enseignants à partir des résultats de leurs élèves, on se doit de constater que les enseignants n'ont, pas davantage qu'en Angleterre, été associés aux changements récents et des interrogations relatives à la déprofessionnalisation du corps enseignant ont également été formulées (cf. Cattonar et Maroy 2000). Au contraire, les enseignants subissent pour la plupart les évolutions en cours et en particulier la redéfinition de leur métier qui transparaît à travers les lignes des différentes réformes: un praticien réflexif, travaillant davantage avec ses collègues et centré sur l'élève-apprenant plutôt que sur la transmission d'un contenu d'enseignement. L'accroissement des règles bureaucratiques évoqué ci-dessus est par ailleurs perçu comme une forme de contrôle, contraire aux discours sur l'autonomie des enseignants. Et le poids du quasi-marché est bien présent. Mais, contrairement au modèle anglais où la présence d'épreuves standardisées tend à homogénéiser les offres scolaires en concurrence, la dynamique présente en Belgique francophone est plutôt propice à la multiplication de niches éducatives (Dupriez et al., 2003): chaque école tend à se spécialiser au regard d'un certain type de public et d'une spécificité dans son offre pédagogique et culturelle. Quoi qu'il en soit, l'expression individuelle et/ou collective des enseignants reste bien faible au regard du poids du marché et d'un recours plus intensif que précédemment à la norme bureaucratique.

Un des points les plus problématiques de l'évolution du pilotage dans ces deux systèmes scolaires, c'est donc bien la place qui est faite aux enseignants comme corps professionnel, susceptible de participer aux délibérations sur le contenu de son métier et les conditions de son exercice. Dans les deux cas évoqués, la tendance à l'harmonisation s'est construite sur la base d'un renforcement de l'autorité politique centrale, parallèlement au développement d'une dynamique de quasi-marché scolaire, dont la signification et l'histoire varient en fonction des pays. Mais, dans les deux cas, la place et la reconnaissance des compétences professionnelles des enseignants est faible. Le défi majeur qui semble se poser est la place des enseignants comme corps professionnel dans un contexte de pilotage par les résultats. D'un point de vue théorique, cette place peut cependant être envisagée et valorisée, même si elle doit se construire contre et au détriment des deux autres sources de légitimité. Elle passe probablement par une plus grande implication des enseignants et de leurs représentants dans la définition des objectifs qui serviront ensuite de référence. Elle passe probablement 
aussi par la mise en œuvre de dispositifs participatifs d'évaluation des établissements où les enseignants ne sont pas seulement soumis à un regard externe, mais participent activement à la construction d'une réflexion sur la qualité de leur travail. Comme l'évoquent notamment Duran et Monnier (1992) ou encore Demailly et al. (1998), une évaluation efficace est nécessairement participative et passe par un travail réflexif des acteurs invités à s'interroger sur leurs pratiques professionnelles. Dans un tel contexte, la mesure des acquis scolaires des élèves idéalement en termes de savoir ajouté - devrait apparaître non pas comme la vérité en soi, mais comme une information parmi d'autres qui vient alimenter l'auto-analyse et l'hétéro-analyse des établissements.

\section{Notes}

1 Le rapprochement que nous opérons entre la logique d'organisation (qu'il est légitime de positionner dans le coin supérieur gauche de la figure) et les établissements mériterait pourtant d'être discutée. A certains moments, et en fonction du contexte, les établissements se rapprochent d'autres formes de coordination: nos propres recherches illustrent par exemple la logique communautaire fortement présente au sein de certains établissements.

2 Jusque fin 1987, on peut encore parler en Belgique de système scolaire national, même si l'administration est séparée pour la gestion des parties francophone et flamande du pays. Depuis 1988, chaque communauté culturelle et linguistique est responsable de son système scolaire et des évolutions contrastées peuvent être observées entre les parties francophone et flamande.

3 A vrai dire, les mauvais résultats des élèves belges francophones lors de l'enquête Pisa 2000 ont eu tendance à accélérer certaines dynamiques déjà présentes et aujourd'hui, les discussions relatives au recours obligatoire à une épreuve standardisée en fin d'école primaire vont bon train.

\section{Bibliographie}

Alter, N. (2001). Linnovation ordinaire. Paris: PUF.

Broadfoot, P. (1996). Education assessment and society. A sociological analysis. Buckingham, Philadelphia: Open University Press.

Broadfoot, P. (2000). Un nouveau mode de régulation dans un système décentralisé: l'Etat évaluateur. Revue française de Pédagogie, 43-55.

Cattonar, B., \& Maroy, C. (2000). Rhétorique du changement du métier d'enseignant et stratégie de transformation de l'institution scolaire. Education et Sociétés, 21-42.

Clarke, P. Feeling compromised: the impact on teachers of the performance culture. Improving schools, 4.

Crahay, M. (1997). Une école de qualité pour tous! Bruxelles: Labor.

De Landsheere, G. (1994). Le pilotage des systèmes d'éducation. Bruxelles: De Boeck université.

Demailly, L., Deubel, P., Gadrey, N., \& Verdière, J. (1998). Evaluer les établissements scolaires: enjeux, expériences, débats. Paris: L'Harmattan.

Derouet, J.-L. (1992). Ecole et justice. Paris: Métailié.

Draelants, H., Dupriez, V., \& Maroy, C. (2003). Le système scolaire en Communauté française. Dossiers du Crisp, $n^{\circ}$ 59, 3-116.

Dupriez, V., Cornet, J., Bodson, X., \& De Smet, N. (2003). La formation des classes et la gestion de l'hétérogénéité à l'école primaire. Rapport de recherche déposé auprès du Ministre de l'Enfance. 
Dupriez, V., \& Maroy Christian. (2003). Regulation in school systems: a theoretical analysis of the structural framework of the school system in French-speaking Belgium. Journal of Education Policy, 18, 375-392.

Duran, P., \& Monnier, E. (1992). Le développement de l'évaluation en France. Nécessités techniques et exigences politiques. Revue Française de Sciences Politiques, 235-262.

Gibson, A., \& Asthana, S. (1999). Schools, markets and equity; access to secondary education in England and Wales. Montréal: Congrès de l'American Educational research Association.

Hollingsworth, J.R., \& Boyer, R. (1997). Coordination of economic actors and social systems of production. In J.R. Hollingsworth \& R. Boyer (Eds.), Contemporary capitalism. The embededdness of institutions. Cambridge: Cambridge University Press.

Jobert, B. (1992). Représentations sociales, Controverses et débats dans la conduite des politiques publiques. Revue française des sciences politiques, 42, 219-233.

Maroy C. (2004). L'impact du décret missions sur les modes de régulation du système d'enseignement: discours et évolutions effectives. In M. Frenay \& C. Maroy (Eds.), L'école, six ans après le décret missions. Regards interdisciplinaires sur les politiques scolaires en Communauté française de Belgique. Louvain-la-Neuve: Presses universitaires de Louvain.

Maroy, C., \& Dupriez, V. ( 2000). La régulation dans les systèmes scolaires. Proposition théorique et analyse du cadre structurel en Belgique francophone. Revue Française de Pédagogie, $130,73-87$.

Neave, G. (1989). On the cultivation of quality, efficiency and enterprise: an overview of recent trends in higher education in Western Europe, 1986-1988. European Journal of Education, 7-23.

Noden, P. (2000). Rediscovering the impact of marketization: dimensions of social segregation in England's secondary schools. British Journal of sociology of education, 21, 371-390.

Pelletier, G. (2001). Décentralisation, régulation et gouvernance des systèmes éducatifs: un cadre de référence. In G. Peletier (Ed.), Autonomie et décentralisation en éducation: entre projet et évaluation. Montréal: Editions de l'AFIDES.

Van Haecht, A. (1998). Les politiques éducatives, figures exemplaires des politiques publiques. Education et Sociétés, 1, 21-46.

Van Zanten, A. (2001). L'inspection des établissements et ses effets sur l'évolution du système scolaire en Angleterre. Entretien avec un responsable de l'OFSTED. Education et Sociétés, 2, 125-134.

Vandenberghe, V. (2002). L'enseignement: état des lieux et utopie. Bruxelles: Labor.

Vandenberghe, V. (1999). Combining market and bureaucratic control in education: an answer to market and bureaucratic failure? Comparative Education, 35, 271-282.

Wrigley, T. (2003). Is school effectiveness anti-democratic? British Journal of Educational Studies, 51, 89-112.

Mots clés: gouvernance, évaluation, régulation, légitimité

\section{Regulierung und Steuerung des Schulsystems: Verfahren der Harmonisierung, aufgezeigt an den historisch gewachsenen, dezentralistisch organisierten Schulsystemen Englands und Belgiens (französischsprachiger Landesteil)}

\section{Zusammenfassung}

Die Schulsysteme Englands und des französischsprachigen Teil Belgiens haben in den vergangenen 15 Jahren einen zentralen Harmionisierungs- und Standardisierungsprozess erlebt. Ein Vergleich der beiden Systeme zeigt auf, wo in den 
unterschiedlichen institutionellen Kontexten damit verbundene Tranformationen umgesetzt werden und wie unterschiedlich auf Evaluation als Ressource zur Steuerung zurückgegriffen wird. Gemeinsam ist beiden Kontexten, dass ersichtlich wird, dass durch die Harmonisierung des Schulsystems der verbindliche Charakter und die Durchsetzungskraft der Standards zunimmt, während gleichzeitig die Professionalität und die Expertise von Lehrpersonen an Gewicht verliert und somit delegitimiert wird.

Schlagworte: Steuerung, Evaluation, Regulierung, Legitimierung / De-Legitimierung

Regolazione e pilotaggio dei sistemi formativi:

I'armonizzazione di due sistemi storicamente decentralizzati, I’Inghilterra e il Belgio francofono

\section{Riassunto}

Negli ultimi 15 anni i sistemi formativi in Inghilterra e nel Belgio francofono hanno attuato un importante processo di armonizzazione e standardizzazione. Il confronto fra i due sistemi mette in evidenza fino a che punto queste trasformazioni avvengano all'interno di contesti istituzionali caratterizzati da tensioni e con delle differenze molto pronunciate nel ricorso alla valutazione quale strumento di pilotaggio. Tuttavia in entrambe le situazioni la chiara avanzata di parametri nazionali prescrittivi tesi all'armonizzazione del sistema sembrano mettere in discussione la legittimità professionale e la competenza degli insegnanti quale fonte di influenza e di riconoscimento.

Parole chiave: valutazione, regolazione, legittimità

\section{Regulation and governance of school systems: towards harmonization in two historically decentralised school systems, England and French speaking Belgium}

\footnotetext{
Summary

The school systems of England and French speaking Belgium have both gone through a process of harmonization and standardization throughout the past 15 years. The comparison of these two systems reveals how much the characteristics of these transformations are related to differences in the contexts. Also, the assessments for students and schools are considerably different. As a similarity appears, however, that the increase in harmonization and use of standards seems to lead to a decrease of recognition of teachers as legitimate professionals and experts, and therefore undermines their influence.
}

Key words: governance, assessment, regulation, legitimacy 